\title{
Les racines de FADIO : généalogie de la formation à distance dans l'Est-du-Québec
}

\author{
The roots of FADIO: genealogy of distance education in Eastern \\ Quebec
}

\section{Las raíces de FADIO: la genealogía de la educación a distancia en el Este de Quebec}

Francis Bastien, doctorant

Université du Québec à Rimouski (UQAR), Canada

francis bastien@uqar.ca

RÉSUMÉ

Cet article propose une rétrospective sur des moments clés de l'histoire de la formation à distance dans l'Est-du-Québec menant à la fondation du projet de Formation à distance interordres (FADIO) et à son évolution récente en contexte de COVID-19. II s'agit ici de retracer, en commençant par les premières itérations de formation à distance (FAD) dans la région, la fondation ou le virage vers la FAD d'établissements d'enseignement. Les caractéristiques de la FAD dans l'Est-du-Québec, les besoins régionaux auxquels elle répond et la clé de son succès seront ainsi abordés à travers le phénomène récurrent de la collaboration entre les établissements.

Mots-clés : FADIO, Est-du-Québec, Bas-Saint-Laurent, Gaspésie-Îles-de-la-Madeleine, histoire, formation à distance

\section{ABSTRACT}

This article offers a retrospective on key moments in the history of distance education in Eastern Quebec leading to the beginning of the project Formation à distance interordres (FADIO) and its recent evolution in the context of COVID-19. This overview aims to retrace, through the first iterations of distance education (DA) in the region, the foundation or the shift towards DA of educational institutions. The characteristics of the FAD in Eastern Quebec, the 
regional needs to which it meets and the key to its success will be examined through the recurring phenomenon of collaboration between institutions.

Keywords: FADIO, Eastern Quebec, Bas-Saint-Laurent, Gaspésie-Îles-de-la-Madeleine, history, distance education

\section{RESUMEN}

Este artículo ofrece una retrospectiva de los momentos clave en la historia de la formación a distancia en la zona de Est-du-Québec, que llevaron a la creación del proyecto "Formation à distance interordres" (FADIO), así como de su evolución reciente en el contexto de COVID19. El objetivo aquí es rastrear, a través de las primeras iteraciones de la formación a distancia (FAD) en la región, la creación o el giro hacia la FAD de las instituciones educativas, así como la colaboración entre instituciones, las características de la FAD en Est-du-Québec, las necesidades regionales que satisface y la clave de su éxito.

Palabras claves: FADIO, Est-du-Québec, Bas-Saint-Laurent, Gaspésie-Îles-de-laMadeleine, historia, formación a distancia

\section{Introduction}

Le vaste territoire du Bas-Saint-Laurent, de la Gaspésie et des Îles-de-la-Madeleine héberge une université, cinq cégeps, quatre instituts et un grand nombre d'écoles primaires et secondaires, confrontés presque tous au défi commun de desservir une population étudiante éparse et limitée par rapport aux établissements urbains. Ce que les cours par correspondance rendaient déjà possible au début du siècle, soit l'accès à une éducation supérieure jusqu'aux confins de régions éloignées, est aujourd'hui un service que certains d'entre nous tiennent pour acquis et dont les exigences de qualité, du côté des professeurs comme des étudiants, ont radicalement augmenté, s'approchant de celles des cours en présence. Le progrès exponentiel des technologies de l'information y contribua, mais il serait réducteur de leur attribuer tout le mérite pour le succès de la formation à distance (FAD), a fortiori sur un territoire où son implantation, son organisation et son développement reposent sur des ressources humaines et matérielles fragmentées.

Comprendre la valeur et le rôle actuels de la FAD dans l'Est-du-Québec demande de remettre en perspective les moments clés de son histoire. II faut retracer, d'une décennie à l'autre, le fil conducteur entre les défis récurrents du système d'éducation dans l'Est-du-Québec, et reconnaître, parmi leurs itérations successives, comment les solutions proposées ont mené à une collaboration unique dans le regroupement de Formation à distance interordres (FADIO), qui constitue aujourd'hui une structure de gouvernance collaborative réunissant les établissements scolaires de tous les ordres d'enseignement du Bas-Saint-Laurent et de la Gaspésie-Îles-de-la-Madeleine par le biais d'une table de partenaires et d'un comité de liaison, telle que schématisée à la figure 1 . 


\section{Figure 1}

Structure de gouvernance de FADIO

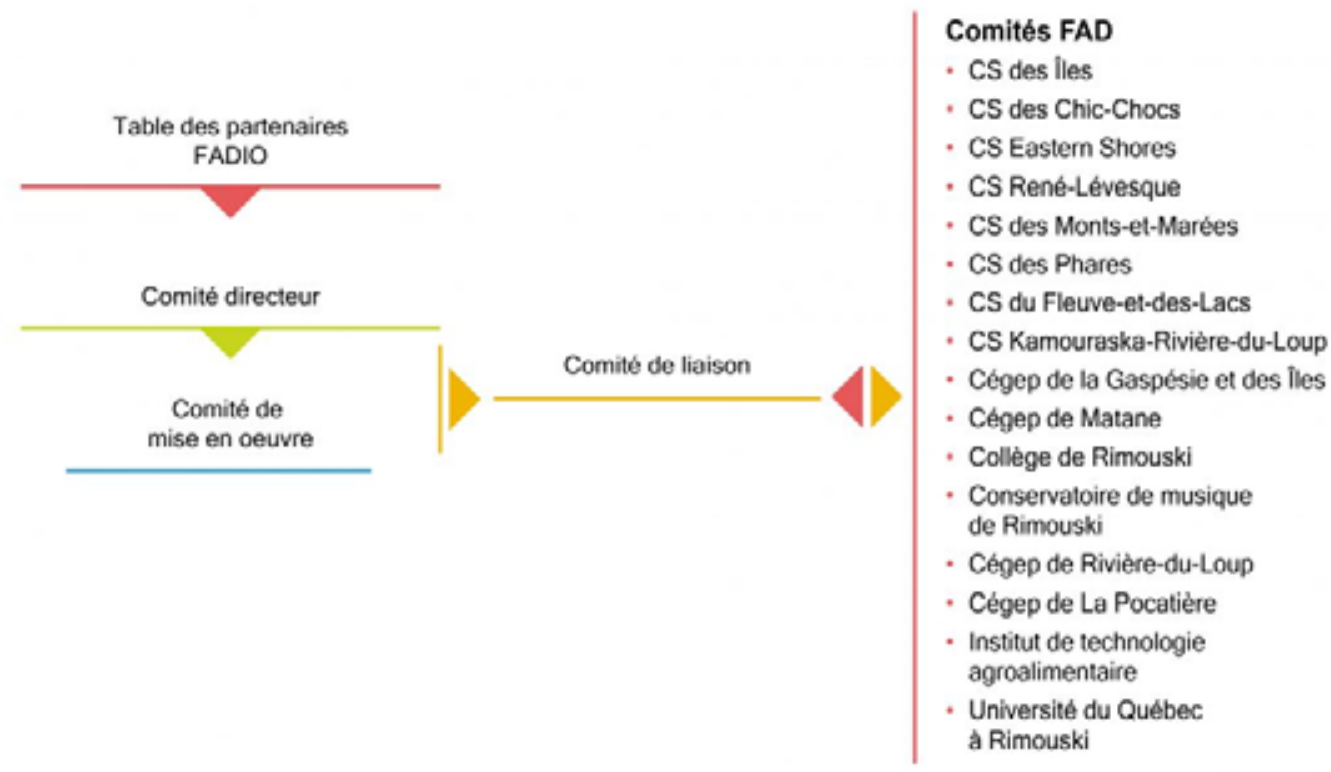

Note. Source : FADIO (s. d.-a)

L'enjeu de pratique au cœur de cette rétrospective consiste en une recherche de solutions en FAD pour répondre aux défis de la baisse démographique, de la distance géographique et des ressources limitées pour le milieu de l'éducation de ces régions. Selon l'Institut de la statistique du Québec (2019, p. 32), « les quatre régions les plus à l'est du Québec [Saguenay-Lac-Saint-Jean, Bas-Saint-Laurent, Gaspésie-lîlesde-la-Madeleine et Côte-Nord] pourraient compter moins d'habitants en 2041 qu'en 2016 ", projection dont les pourcentages, indiqués à la figure 2 , surpassent ceux de l'édition précédente et peuvent difficilement être ignorés par les établissements scolaires. 
Figure 2

Variation projetée de la population totale, scénario Référence (A), Québec, régions administratives et régions métropolitaines de recensement (RMR), 2016-2041

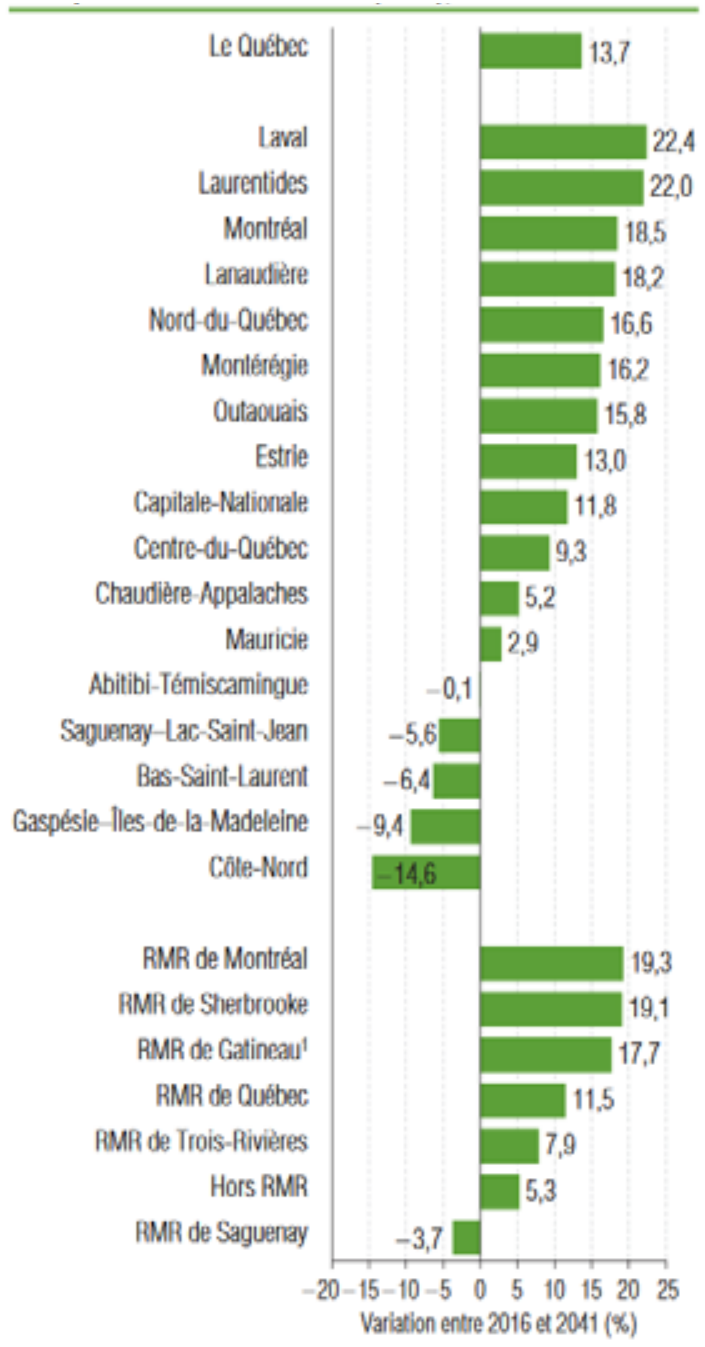

Note. Source : Institut de la statistique du Québec (2019, p. 32)

Déjà en 2004, le constat du Centre facilitant la recherche et l'innovation dans les organisations (CEFRIO) vis-à-vis de ces enjeux dans le milieu collégial soulignait que « les moyens de consolidation actuellement mis en œuvre ont atteint leurs limites et qu'il faudrait donc envisager sérieusement la mise sur pied d'une autre stratégie, celle du recours aux technologies de l'information et de la communication » (CEFRIO, 2004 , p. 5). Plus qu'une mesure transitoire ou palliative, une exigence s'imposait de réorganiser à long terme la gestion des cégeps. Or, l'existence de compétences et d'infrastructures de FAD disséminées sur le territoire ne suffisant pas pour surmonter ces défis complexes dans un contexte où la survie de petits établissements est menacée par la pénurie de ressources, la collaboration interinstitutionnelle joua et joue un rôle essentiel dans la mise en œuvre de ces stratégies, comme cherche à le démontrer l'examen historique qui suit. 


\section{Les débuts de la FAD dans l'Est-du-Québec}

En 1968, un reportage de Radio-Canada soulignait l'innovation du téléscript, technologie qui permettait pour la première fois aux instituteurs des villes d'être entendus par voie téléphonique, alors même que leur écriture était transmise et projetée en direct devant toutes les classes reliées au réseau de téléscript du Bas-Saint-Laurent (ICl Bas-Saint-Laurent, 2020, 20 août). La communication directe, combinée au support visuel du projecteur, offrait quelque chose de réellement novateur : l'ébauche d'un sentiment de présence et d'interaction. Rejoignaient les étudiants de chaque village, non seulement la matière, mais aussi la voix et la démonstration écrite du professeur, malgré la petitesse des groupes et le manque de personnel qui, en d'autres circonstances, auraient forcé les étudiants à se déplacer. Le réseau, technologique autant qu'humain qui se tissait entre les établissements d'enseignement des villes et les écoles de village, continuera de s'étendre et de se solidifier. À cet égard, le reportage de 1968 ne témoigne pas que d'une admiration d'époque devant les « merveilles de la science ", mais révèle surtout le prototype d'une solidarité régionale en éducation, dont la technologie était le catalyseur.

\section{Le virage vers la formation à distance}

Malgré cette percée, le coût et la portée des technologies posaient encore des limites considérables. Pour cette raison, la FAD restera longtemps marginale dans son ampleur comme dans la priorité qu'elle représente au sein des établissements. II faudra attendre l'arrivée puis la démocratisation d'Internet pour que les barrières technologiques soient radicalement diminuées et que se présente l'opportunité de bâtir un réseau à très grande échelle. À ses débuts, la FAD était souvent considérée comme une extension de la formation continue, un service complémentaire se greffant aux programmes de cours présentiels, ce qui explique pourquoi beaucoup d'établissements gardent des traces de ce fonctionnement plus localisé dans leur gestion. Peu à peu, la transition vers ce mode d'enseignement engendra la création de nouveaux organismes et de nouveaux partenariats, dont la gestion s'adapte à des besoins différents.

\section{Développement d'une expertise en région}

Desservir adéquatement un territoire aussi vaste que le Bas-Saint-Laurent ou la Gaspésie requiert avant tout une capacité d'adaptation et de collaboration. II n'est plus suffisant de se munir d'une structure éprouvée sur le modèle des grandes universités, car tous les acteurs - enseignants, gestionnaires, conseillers pédagogiques - et toutes les ressources matérielles et financières ne se retrouvent pas sous le même toit. Pour bonifier l'offre limitée des cours à distance à l'Université du Québec à Rimouski (UQAR) comme dans les cégeps, qu'ils soient synchrones, asynchrones ou hybrides, il ne fallait pas seulement développer une compétence, mais aussi en amplifier le rayonnement et en simplifier l'accès pour les étudiants intéressés. Et dans une région où aucun établissement ne dispose des ressources suffisantes pour atteindre seul tous ces objectifs, une plus grande collaboration s'imposa.

La trajectoire du consortium Collegia est un bon exemple d'adaptation motivée par ce besoin. En 2000, les services de formation continue du Cégep de la Gaspésie et des îles, du Cégep de Matane et du Cégep de Rivière-du-Loup formèrent ce consortium afin de mutualiser leurs ressources. II ne s'agissait pas encore de FAD, mais d'une expertise en « ingénierie de formation » mobilisée dans des projets internationaux de formation continue et d'accompagnement des enseignants. Le virage vers la $F A D$, facilité par ce partage de ressources, s'accentua au Cégep de Matane en 2004 dans la continuité du projet « Les cégeps en réseau » qui visait entre autres la consolidation de l'offre de cours dans certains programmes à petites cohortes (CEFRIO, 2004). La fusion des groupes de différents cégeps au sein d'un même cours en ligne offert en mode synchrone par l'un ou l'autre établissement, lequel aurait manqué d'inscriptions en temps 
normal, permit de garantir une continuité et une offre de cours suffisante dans le parcours des étudiants. Aujourd'hui, Collegia se fait connaître pour son expertise en formation à distance autant qu'en présence dans les cégeps, dans les entreprises et à l'étranger, témoignant d'une approche à géométrie variable qui semble répondre plus adroitement, désormais, aux exigences d'une réalité en constant changement.

L'Eastern Shore School Board (ESSB) est une commission scolaire dont l'expérience en FAD remonte à plus loin encore et dans laquelle on reconnaît une adaptation similaire. Née de la fusion en 1998 des commissions scolaires anglophones de la Gaspésie, des Îles-de-la-Madeleine, du Bas-Saint-Laurent et de la Côte-Nord, l'ESSB représente un bassin d'étudiants anglophones, aux effectifs en déclin constant depuis l'entrée en vigueur de la loi 101'1, et dispersés sur un immense territoire (Journeau, 2008). II n'est donc pas étonnant que le besoin de développer la FAD se soit imposé là aussi comme mesure de survie bien avant que FADIO en arrive au stade de projet et que l'ESSB se joigne à l'initiative en 2017. Déjà en 1997, Andy Ross, enseignant à l'école d'Escuminac, offrait le premier cours crédité de niveau secondaire entièrement en ligne, un défi impressionnant d'autant plus qu'il s'assura de maximiser l'interaction avec les élèves grâce aux moyens dont il disposait : présentation affichée à l'écran, voix transmise en direct, exercices tracés à l'ordinateur par les élèves à l'aide d'un crayon tactile (ESSB, s. d.). Encore aujourd'hui, la FAD offre un filet essentiel pour relier et soutenir les élèves et les enseignants de la commission scolaire.

\section{L'enseignement à distance à l'UQAR}

L'UQAR demeure le plus grand établissement d'enseignement supérieur de l'Est-du-Québec et offre des services à distance depuis ses débuts. "Dans les années 80, des cassettes et des livres étaient postés aux personnes inscrites aux cours "médiatisés" » nous rappelle le numéro spécial de l'Universitaire publié à l'occasion du 50 ${ }^{\circ}$ de l'UQAR (UQAR, 2019, p. 27). Le virage marqué vers la FAD se fit dans les années 90 avec le début des cours à distance, d'abord asynchrones puis, dans la deuxième moitié des années 90 , synchrones, utilisant pour la première fois la vidéoconférence. Cet ajout visait d'abord à contribuer au développement de la région et à la formation continue des travailleurs et des professionnels en entreprise, mais aussi à répondre au besoin criant de formation des maîtres dans la région afin qu'un plus grand nombre d'enseignants obtiennent leur diplôme. II n'est donc pas étonnant qu'en 2013 I'UQAR ait lancé le baccalauréat en enseignement professionnel à titre de premier baccalauréat entièrement à distance. Au cours de ces deux décennies, les moyens technologiques firent des progrès considérables, avec l'apparition des environnements numériques comme Claroline, qui fut employé dans les années 2000, puis Moodle, aujourd'hui le support bien connu du Portail étudiant (UQAR, 2019, p. 27).

Les trajectoires tracées par l'évolution de ces instances et des organisations mentionnées plus haut, aussi disjointes qu'elles puissent paraître à cette étape, se rapprochaient déjà par leurs défis communs et leur reconnaissance du rôle de la FAD - convergence qui laissait entrevoir la possibilité, et bientôt la nécessité, d'une association à plus grande échelle.

\section{Naissance et évolution de FADIO}

Au début des années 2010, dans le contexte des coupures du gouvernement de l'époque en éducation (qui culmineront avec la fermeture des directions régionales du ministère de l'Éducation du Québec en 2014) et d'une baisse démographique non négligeable, la pression pour réorganiser le réseau scolaire des régions s'ajouta à celle, déjà forte, pour y développer la FAD. II s'agissait de faire face à la compétition, très attirante pour les jeunes du Bas-Saint-Laurent ou de la Gaspésie, des cégeps et des universités de

\footnotetext{
${ }^{1}$ La loi 101, ou Charte de la langue française, adoptée en 1977, affirme le français comme langue officielle du Québec et définit les droits linguistiques des Québécois.
} 
Québec ou de Montréal, après qu'Internet ait presque aboli les barrières géographiques. C'est pourquoi en 2012, à l'initiative du Cégep de Matane, une étude exploratoire sonda les établissements d'enseignement du Bas-Saint-Laurent et de la Gaspésie-Îles-de-la-Madeleine dans le but d'identifier les besoins et d'esquisser le meilleur mode de fonctionnement d'une éventuelle association.

\section{Exploration et vecteurs communs}

Avant la formation de FADIO, dont la première entente remonte à $2013^{2}$, la Table interordres d'éducation du Bas-Saint-Laurent (TIO BSL) et la Table Éducation-Formation-Emploi de la région Gaspésie-liles-dela-Madeleine (TÉFE GîM) réunissaient des responsables de tous les niveaux d'enseignement, mais la gestion se faisait plus localement. L'étude exploratoire lancée en 2012 avait donc pour objectif de " prendre le pouls de ces établissements et d'identifier des vecteurs communs » en vue d'une collaboration plus étroite en FAD, explique Daniel LaBillois, responsable du Centre de pédagogie universitaire à l'UQAR et collaborateur de FADIO depuis sa création (communication personnelle, 5 novembre 2020).

Le constat apparut clairement. Pour atteindre et préserver l'excellence, il faudrait maintenir un dialogue constant, un partage des ressources et une transmission des compétences à échelle interordre : tous les établissements scolaires des deux grandes régions pouvaient contribuer et avaient à gagner d'une telle entente (Simard, 2013). La mise en place d'un partenariat relevait de la nécessité, mais cela ne signifiait pas que les membres s'y joindraient à contrecœur. Au contraire, même si l'existence de programmes similaires d'un établissement à l'autre pouvait engendrer une certaine concurrence, la véritable compétition se trouvait dans les grandes villes. Pour contrer la concurrence et du même coup assurer l'accessibilité et la viabilité des programmes de formation, les partenaires locaux, soit I'UQAR et l'ensemble des commissions scolaires, cégeps et instituts du Bas-Saint-Laurent et de la Gaspésie-Îles-de-la-Madeleine allaient s'allier sur deux vecteurs fondamentaux, soit la pédagogie et la technologie.

\section{Le point de bascule}

La transition du mode de gestion par les Tables régionales interordres à celui de FADIO, impliquant une coopération plus directe et une table des partenaires unique, ne se fit pas du jour au lendemain, bien qu'elle constitue un point charnière. Pour Mylène Simard, coordonnatrice de FADIO, cela représentait un enjeu de mise en commun des ressources pour former les enseignants, prévoir des achats groupés et s'ajuster plus agilement aux changements nécessaires (Radio-Canada, 2017). Le plan d'action visait entre autres à garantir la "disponibilité de la meilleure ressource sur le territoire », pour tous les membres, chaque fois qu'un nouveau défi émergeait. S'ajouta le souci de " pérenniser la structure » en assurant la rotation des responsables, la documentation et la transmission continue des compétences (D. LaBillois, communication personnelle, 5 novembre 2020). À long terme, ce principe de partage équitable, le respect mutuel et la reconnaissance du fait que chaque établissement a besoin des autres pour atteindre ses propres objectifs dans un domaine aussi multidisciplinaire, inspirèrent la confiance et un sentiment d'appartenance - et motivèrent les membres à s'engager dans une gouvernance collaborative.

\footnotetext{
${ }^{2}$ Cette première entente réunissait l'UQAR, les commissions scolaires, les cégeps et les instituts du Bas-Saint-Laurent et de la Gaspésie-îlesde-la-Madeleine figurant dans la structure de gouvernance actuelle de FADIO (figure 2), à l'exception de l'ESSB et du Conservatoire de musique de Rimouski, qui rejoindront FADIO en 2017.
} 
La première rencontre des partenaires, qui eut lieu en juin 2014, fut l'occasion pour tous les membres de faire un bilan des premiers mois, de présenter leurs contributions et leurs réussites. L'échange non seulement confirma la raison d'être de FADIO, mais renforça le désir de chacun d'y participer, menant jusqu'au renouvellement de l'entente de collaboration en 2017, puis en 2020.

\section{La crise pandémique}

La situation exceptionnelle du confinement engendrée par la pandémie de COVID-19 aurait sans doute eu des répercussions plus néfastes sur l'expérience des étudiants et la tâche des acteurs du milieu de l'enseignement si le réseau n'avait pas réaffirmé sa cohésion et atteint un niveau supérieur de flexibilité et de transférabilité des compétences. Outre la structure de gouvernance horizontale, des projets mis en branle par FADIO bien avant la pandémie jettent aujourd'hui la base d'un système robuste d'accompagnement pour tous ses membres. Soutien FADIO, notamment, un projet qui devait initialement prendre la forme de camps et d'ateliers ponctuels, a pris de l'ampleur jusqu'à devenir une plateforme permanente, sur Internet ou par ligne téléphonique d'urgence, où les formateurs peuvent recevoir gratuitement un soutien technopédagogique de deuxième ligne (FADIO, s. d.-b). À ce service, offert par différents conseillers pédagogiques, technopédagogues et spécialistes sur le territoire, s'ajoute l'animation d'ateliers exploratoires sur les plateformes Microsoft Teams ou Moodle.

Comme l'explique Mylène Simard (communication personnelle, 16 novembre 2020) : « Pour répondre au nombre important d'acteurs de l'éducation à la recherche de solutions pour adapter leur pratique à la FAD, on a multiplié la mise en place de communautés de pratique et d'apprentissage, favorisant ainsi le partage d'expertise. " Les établissements n'échappèrent pas à la crise, mais eurent promptement accès à ces recours, qu'il aurait été ardu de mobiliser sans partenariat, contribuant à accroître leur autonomie.

\section{Discussion}

Le bilan de ces évènements notables dans l'histoire de la FAD dans l'Est-du-Québec, condensés sous forme de ligne du temps sommaire à la figure 3, fait apparaître des constats qui font écho aux préoccupations ayant mené à la création de FADIO : d'une part, le rôle central de la FAD pour affronter les enjeux de baisse démographique et de ressources limitées et, d'autre part, le besoin de façonner des structures de gouvernance collaborative pour assurer la survie des petits établissements sur un immense territoire à desservir. 
Figure 3

Quelques jalons de l'histoire de FADIO et de la FAD dans l'Est-du-Québec projetée

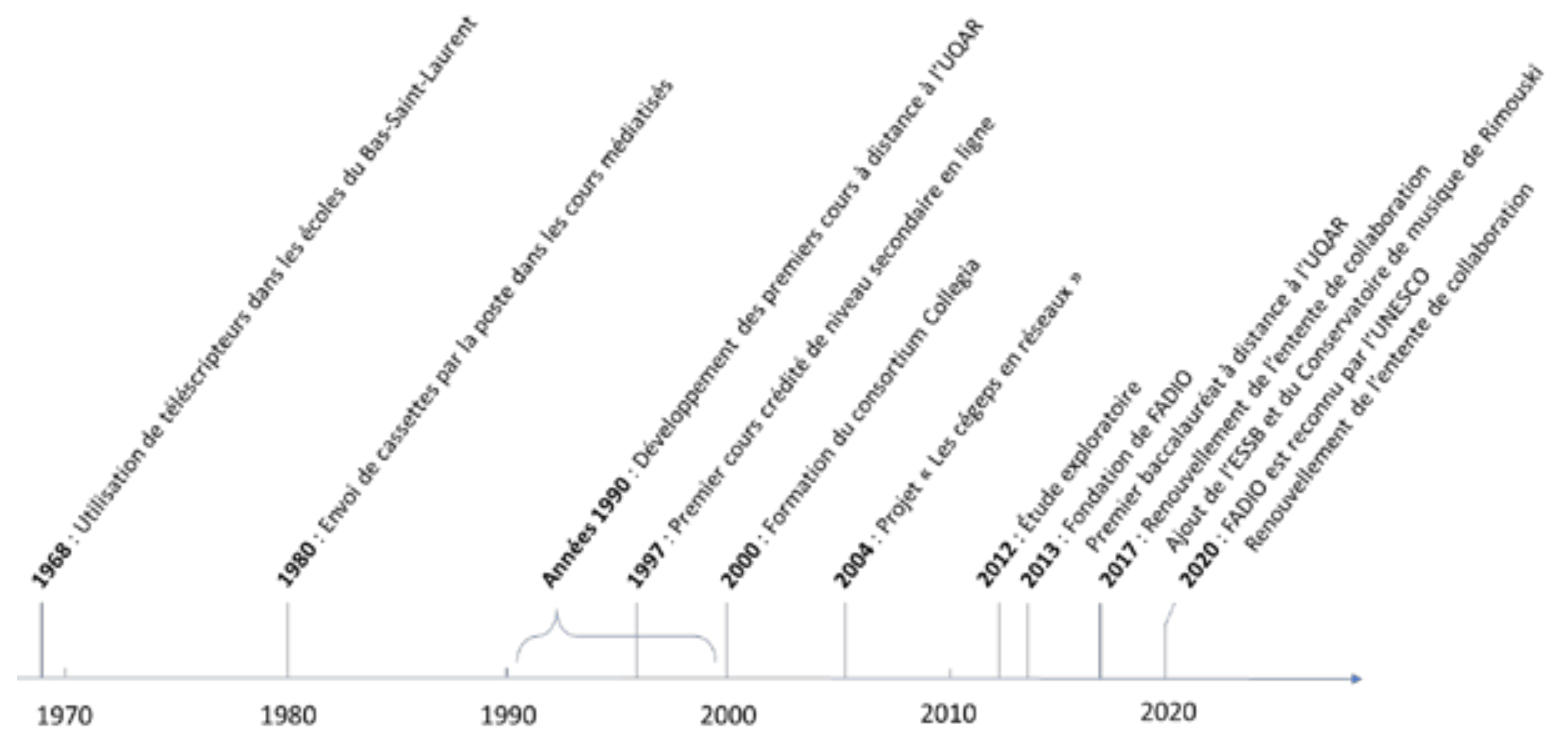

L'efficacité des pratiques de FADIO fait régulièrement l'objet d'une évaluation interne, entre autres par son comité de qualité de la FAD, dont le rôle est de produire des outils servant de cadre de référence pour le développement de la FAD et par le biais d'un questionnaire adressé aux membres chaque année permettant de mesurer l'atteinte d'une série d'objectifs fixés par le plan d'action annuel (M. Simard, 5 février 2021, communication personnelle). L'épreuve de la pandémie mit en lumière les forces de ce modèle, la rapidité d'action, la flexibilité, la qualité du soutien offert, mais occasionna également un test révélateur de ses limites, notamment communicationnelles. Dans un réseau aussi vaste, reliant des milieux séparés par le biais d'un comité de liaison, de professionnels en prêt de services et d'échanges numériques, il suffit qu'un maillon de la chaîne échoue à transmettre l'information pour que l'ensemble de la communication soit compromis. Pour résoudre cette difficulté, FADIO cherche à établir des voies de communication plus directes avec les conseillers pédagogiques et les technopédagogues sur le terrain, en leur offrant simultanément davantage de formation afin de consolider leur autonomie (M. Simard, 5 février 2021, communication personnelle).

\section{Conclusion}

En mai 2020, FADIO reçut de l'UNESCO le titre de « pratique prometteuse en éducation », reconnaissance internationale soulignant une contribution majeure à l'accès à l'éducation pour tous. Parmi les critères de sélection figurent le caractère innovant des méthodes et leur impact sur la communauté, l'engagement des parties prenantes, la gestion compétente, la documentation et l'adaptabilité des pratiques (UNESCOUNEVOC, s. d.). Non seulement cette reconnaissance met en valeur le partenariat dans l'Est-du-Québec, mais «FADIO est la première initiative en Amérique du Nord à être retenue dans ce répertoire qui réunit douze autres pratiques à l'échelle mondiale » (FADIO, 2020).

Ces apports reconnus et éprouvés dans la dernière année - engagement commun, adaptabilité, gestion plus efficiente - ne rendent pas moins substantielles les limites de la structure et les défis qu'elles posent 
dans un avenir rapproché, comme la complexité des voies de communication et de l'organisation au sein d'un réseau aussi étendu. II n'est pas anodin de faire correspondre une gestion à l'échelle humaine qui repose sur le partage et la communication directe entre les acteurs sur le terrain, avec un mode d'enseignement où lesdits acteurs sont de facto séparés par des centaines de kilomètres. S'il s'agit là de la clé du succès de FADIO; on peut aussi y reconnaître un modèle inspirant de développement régional pour l'éducation au Québec.

\section{Liste de références}

CEFRIO. (2004). Projet Les Cégeps en réseau. La mise en réseau de programmes techniques en difficulté peut-elle permettre leur consolidation? https://www.collegeahuntsic.qc.ca/documents/3d01eb37-3232-4925-809d-9c5be7233e7b.pdf

ESSB. (s. d.). Distance Education. Eastern Shores School Board. https://schools.essb.qc.ca/escuminac/our-history/distance-education/

FADIO. (s. d.-a). À propos. https://www.fadio.net/a-propos/

FADIO. (s. d.-b). Besoin de soutien? https://www.fadio.net/soutien/

FADIO. (2020, 12 mai). FADIO reçoit le titre de pratique prometteuse en éducation de l'UNESCO. https://www.fadio.net/fadio-recoit-le-titre-de-pratique-prometteuse-en-education-de-lunesco/

ICI Bas-Saint-Laurent. (2020, 20 août). Canada Magazine, janvier 1968 [vidéo jointe] [statut Facebook]. Facebook. https://www.facebook.com/icibassaintlaurent/videos/3344662548925420

Institut de la statistique du Québec. (2019). Perspectives démographiques du Québec et des régions, $2016-2066$. https://statistique.quebec.ca/en/fichier/perspectives-demographiques-du-quebec-et-des-regions-2016-2066-edition2019.pdf

Journeau, C. (2008). Historical Perspective. Eastern Shores School Board. https://www.essb.qc.ca/plan/en/index.php?page=historical

Radio-Canada. (2017, 15 septembre). FADIO : un programme de formation à distance de grande envergure renouvelé. https://tinyurl.com/82z4sx68

Simard, M. (2013, 26 novembre) Un projet de formation à distance interordres suprarégional et novateur. Portail du réseau collégial du Québec.

http://lescegeps.com/nouvelles/mots-cles/table_interordres_deducation_du_bas_saint_laurent_tio_bsl

UNESCO-UNEVOC. (s. d.). Sélection des pratiques prometteuses. https://unevoc.unesco.org/home/Cadre+conceptuel+pour+l+evaluation+des+pratiques+prometteuses+en+EFTP

Université du Québec à Rimouski (UQAR). (2019). 50 ans de formation à l'UQAR [Numéro spécial]. L'universitaire, 26-27. https://fr.calameo.com/read/000879539931cf2b0f894?page=1 